\title{
Application of a Physical Ensemble Method in the POD-4DEnVar
}

\author{
YUXUAN YANG \\ College of Meteorology and Oceanography, National University of Defense Technology, Nanjing, \\ and Unit 66199, People's Liberation Army, Beijing, China \\ LIFENG ZHANG \\ College of Meteorology and Oceanography, National University of Defense Technology, Nanjing, China \\ BIN ZHANG \\ Unit 61741, People's Liberation Army, Beijing, China \\ WeI You AND MingYANG ZHANG \\ College of Meteorology and Oceanography, National University of Defense Technology, Nanjing, China
}

\section{BINPENG XIE}

College of Meteorology and Oceanography, National University of Defense Technology, Nanjing, and Unit 94783, People's Liberation Army, Huzhou, China

(Manuscript received 22 November 2017, in final form 10 September 2018)

\begin{abstract}
The sensitivity of the proper orthogonal decomposition (POD)-based ensemble four-dimensional variational assimilation (4DVar) method (referred to as POD-4DEnVar) to cumulus and microphysics schemes was investigated using the Weather Research and Forecasting (WRF) Model for heavy rainfall in South China. Results show that the choice of the cumulus and microphysics schemes for ensemble samples significantly impacts precipitation prediction and that Doppler radar data assimilation using POD-4DEnVar is sensitive to the parameterization schemes used for the ensemble samples. The cumulus and microphysics schemes primarily affect the vertical velocity and rainwater mixing ratio of the ensemble forecasts. Variations in the ensemble samples caused by different parameterization schemes are introduced into the fourdimensional ensemble variational assimilation by the radar data observation operator. These variations affect the analysis fields and result in variations in precipitation prediction. To obtain the optimal result (smallest forecast error), three methods are designed based on the physical ensemble technique, which can filter out the effects of different parameterization schemes for the ensemble samples through averaging. The results show that the precipitation forecasts from the three assimilation experiments are improved compared with a control experiment, but each physical ensemble method leads to a unique precipitation forecast.
\end{abstract}

\section{Introduction}

The chaotic nature of the atmosphere means that minor deviations of the initial fields in numerical predictions lead to dramatic changes in the evolution of the simulated atmospheric system (Lorenz 1963). With the gradual improvement of numerical models, the quality of the initial fields has played an increasingly important role in improving the accuracy of numerical weather

Corresponding author: Lifeng Zhang, zhanglif_qxxy@sina.cn forecasts. To provide more accurate initial fields, the introduction of nontraditional data (including satellite and radar data) into numerical models has been widely adopted. Assimilation techniques for these nontraditional data have been rapidly developed. Currently, two general approaches-variational data assimilation methods (3DVar and 4DVar) and ensemble methods [EnKF, ensemble square root filter (EnSRF), etc.]—are widely used in Doppler radar data assimilation (Gao et al. 1999; Snyder and Zhang 2003; Xiao et al. 2007; Zhang et al. 2009). In recent years, ensemble variational 
data assimilation (referred to as EVA; 3DEnVar, 4DEnVar, etc.), which combines the two methods, has also been used (Hamill and Snyder 2000; Buehner 2005; Liu et al. 2008). Compared with the 3DEnVar method, the 4DEnVar method uses the complete numerical model equations as the dynamic constraint. This leads to trajectory patterns that are in better agreement with the observations than is the case for 3DEnVar. Thus, many specific implementation plans based on the 4DEnVar method have been put forward (Qiu and Chou 2006; Tian et al. 2008; Wang et al. 2010). The EVA method adopted in this paper, referred to as POD-4DEnVar, was proposed by Tian et al. $(2008,2009,2011)$ and Tian and Feng (2015) and is based on proper orthogonal decomposition (POD) and ensemble forecasting. This method applies the POD technique to the 4DVar framework, and the observation perturbations are decomposed so as to extract the standard orthogonal basis of the forecast bias in a four-dimensional (4D) space, which can represent the spatial structures and patterns of evolution of the 4D variables. For other traditional 4DEnVar methods, such as the singular value decomposition (SVD) 4DEnVar method (Qiu and Chou 2006), the model and observation perturbations are decomposed together, which needs more computation than the POD-4DEnVar method. This method has also been successfully applied to land data assimilation (Tian et al. 2009, 2010) and Tan-Tracker joint data assimilation (Tian et al. 2014). Also, the POD-4DEnVar-based radar assimilation system on the Weather Research and Forecasting (WRF) Model platform has been built, and significant improvement in precipitation prediction with radar data assimilation has been achieved (Zhang et al. 2015).

Based on the variational data assimilation framework, EVA constructs the flow-dependent background error covariance using ensemble forecast information, thereby improving the quality of the analysis field and numerical predictions. Therefore, high quality ensemble forecast data are a prerequisite for EVA, and the quality of the ensemble forecast is an important factor in determining the quality of EVA (Ma et al. 2014). Many numerical weather prediction (NWP) centers such as the European Centre for Medium-Range Weather Forecasts (ECMWF) and the National Centers for Environmental Prediction (NCEP) can produce high quality ensemble forecasts that improve prediction results from EVA (Buehner et al. 2010a,b). In the current study using EVA, many ensemble forecasts are assembled from several different initial fields with the optimal initial perturbation method. However, it is also necessary when forecasting severe convective systems, such as heavy rainfall in a moist atmosphere, to consider the uncertainty or diversity of the physics process when constructing the ensemble samples. Because of the close relationship between the development of heavy rainfall and physical processes in the atmosphere, the numerical simulations are very sensitive to the parameterization schemes of the model, and uncertainty caused by different parameterization schemes can lead to uncertainty in the simulation results (Meng and Zhang 2007; Lan et al. 2010). As a result, the physical ensemble method is an effective approach for eliminating this uncertainty (Peffers 2011). An ensemble forecasting system, which fully considers uncertainties in the initial fields and parameterization schemes, forms ensemble samples that reflect the atmospheric evolution that occurs under a variety of conditions and thus maximizes the accuracy of EVA.

For the POD-4DEnVar method adopted in this paper, because the observation perturbations are the deviations of the ensemble samples and the background field in the observation space, the assimilation result is also dependent on the quality of the ensemble samples when the background field is given. In this case, it is important to assemble representative ensemble samples in order to obtain an optimal assimilation analysis field and improve the accuracy of the numerical weather predictions. However, the ensemble samples may differ when different cumulus and microphysics schemes are selected, which may further affect the assimilation and numerical forecasts. Therefore, the sensitivity of the POD-4DEnVar method to the parameterization (cumulus and microphysics) schemes of the ensemble samples and how to weaken the influence of the parameterization schemes are worth discussing.

The remainder of this paper is organized as follows. A new radar data assimilation scheme based on the POD-4DEnVar approach and applied to a heavy rainfall event is briefly presented in section 2 . Section 3 explores the influence of the cumulus and microphysics schemes of the ensemble samples on the POD-4DEnVar results. In section 4, three methods based on the physical ensemble techniques, which can weaken the influence of the parameterization schemes, are introduced. Section 5 summarizes the results and discusses potential future improvements.

\section{The POD-4DEnVar method and the heavy rainfall event}

\section{a. Overview of the heavy rainfall event}

On 8-9 December 2015, a heavy rainfall occurred throughout East and South China according to the observed data (http://data.cma.cn/data/detail/dataCode). 


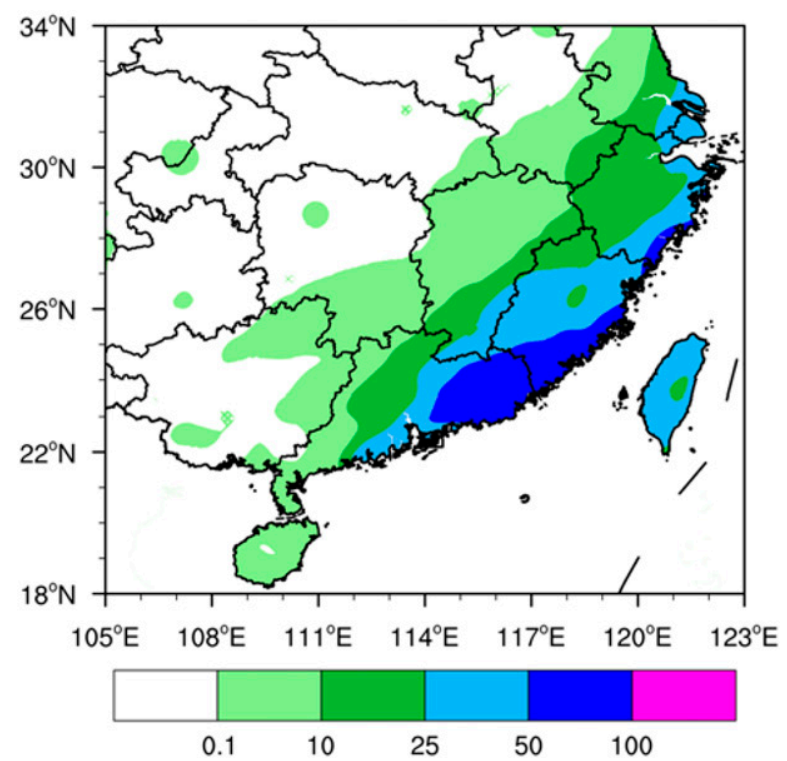

(a)

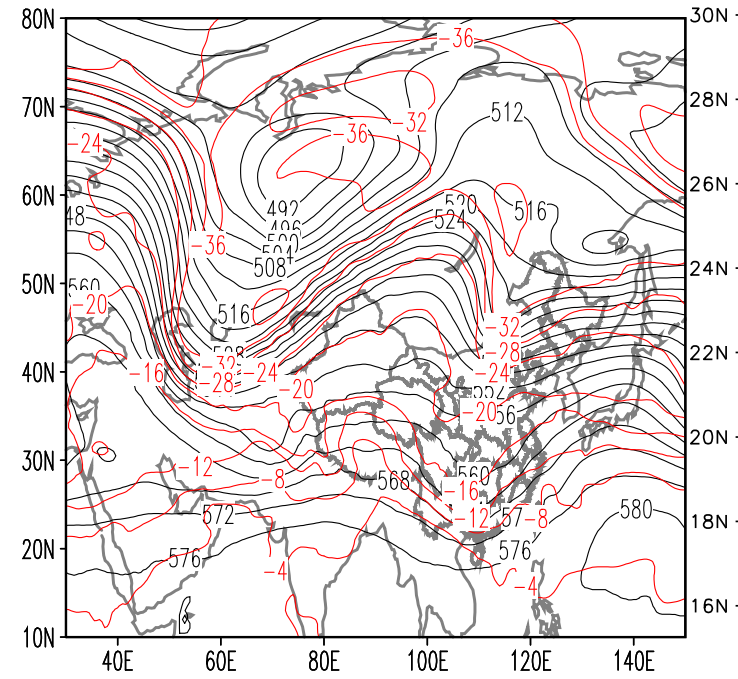

(b)

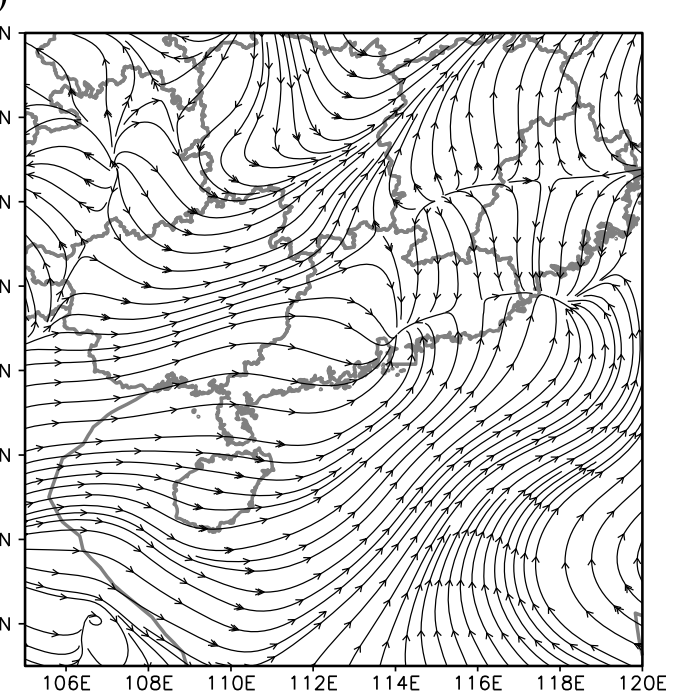

(c)

FIG. 1. (a) The 24-h accumulation precipitation (mm) from 2000 UTC 8 Dec to 2000 UTC 9 Dec 2015. (b) The geopotential (black contour) and temperature at $500 \mathrm{hPa}$ (red contour) and (c) the flow field (streamline) at $850 \mathrm{hPa}$ valid at 1200 UTC 9 Dec 2015.

Eastern Guangdong, southern Fujian, and southeastern Zhejiang Provinces suffered rainstorm-magnitude precipitation (24-h accumulated precipitation greater than $50 \mathrm{~mm}$ ). In some areas, the amount of precipitation exceeded $100 \mathrm{~mm}$ in $24 \mathrm{~h}$, and the coverage and amount of precipitation far surpassed historical levels. The rainfall event lasted from 2000 UTC 8 December to 2000 UTC 9 December 2015 (Fig. 1a) and was heaviest at 1200 UTC 9 December 2015.

The large-scale environment was generally favorable for the development of the heavy rainfall at 1200 UTC
9 December. In the upper levels (Fig. 1b), a moving trough was located near $115^{\circ} \mathrm{E}$, which is close to the area of the heavy rainfall. In accordance with the upper moving trough, a convergence belt was generated near $22^{\circ}-24^{\circ} \mathrm{N}$, $114^{\circ}-118^{\circ} \mathrm{E}$ at $850 \mathrm{hPa}$ (Fig. 1c), which also provided favorable dynamic conditions for the occurrence of the heavy rainfall. A detailed analysis is provided in Yang et al. (2016). Here, the observed geopotential, temperature, and flow field are derived from the $1^{\circ} \times 1^{\circ} \mathrm{NCEP}$ Final (FNL) Operational Model Global Tropospheric Analyses (http://dss:ucar.edu/datasets/ds083.2/matrix.html). 


\section{b. Model configuration}

The Advanced Research version of the WRF (ARW), version 3.6 (Skamarock et al. 2008), is used for this study, and the simulated domains were configured as shown in Fig. 2. Two two-way nested domains are used. The outer domain $(\mathrm{d} 01)$ is on a $200 \times 200$ horizontal grid with a resolution of $15 \mathrm{~km}$, and the inner domain (d02) has a horizontal grid spacing of $5 \mathrm{~km}(433 \times 394)$. Thirty $\eta$ vertical levels up to $50 \mathrm{hPa}$ are used for both domains. Before the EVA experiments, tests of different parameterization combinations have been carried out and the following schemes produce smaller errors in the forecast compared to other parameterization combinations. The WRF single-moment 6-class (WSM6) scheme (Hong and Lim 2006) for microphysics, the Grell-Freitas (GF) scheme (Grell and Freitas 2014) for cumulus convection, the Yonsei University (YSU) scheme (Hong et al. 2006) for the planetary boundary layer, the Noah land surface model (NLSM; Ek et al. 2003) for land surfaces, the Rapid Radiative Transfer Model (RRTM; Mlawer et al. 1997), and the Dudhia scheme (Dudhia 1989) for longand shortwave radiation are used for the forecast rather than the construction of the ensemble samples and the assimilation.

Doppler radar data assimilation is conducted from 1900 UTC 8 December to 2000 UTC 8 December in the inner domain at 6-min intervals. Using the analysis field at 1900 UTC 8 December 2015 as the initial field, the numerical model is integrated for $29 \mathrm{~h}$ in both domains. The $1^{\circ} \times 1^{\circ}$ NCEP FNL Operational Model Global Tropospheric Analyses are utilized as the first-guess field and boundary conditions in the experiments, and the reflectivity and radial velocity from the Chinese Meteorological Administration's (CMA) Meizhou radar, which is located in Guangdong Province $\left(115.99^{\circ} \mathrm{E}\right.$, $24.26^{\circ} \mathrm{N}$; Fig. 2) at an altitude of $416 \mathrm{~m}$, are both used in the assimilation. The radar has nine elevation scans with elevation angles of $0.5^{\circ}, 1.5^{\circ}, 2.4^{\circ}, 3.4^{\circ}, 4.3^{\circ}, 6.0^{\circ}, 9.9^{\circ}$, $14.6^{\circ}$, and $19.5^{\circ}$.

The raw data from the Meizhou radar have substantially high resolution, which is typically updated every 6 min or so with $250-\mathrm{m}$ resolution for radial velocity and $1-\mathrm{km}$ resolution for reflectivity. To utilize the radar data effectively, several data preprocessing procedures, including denoising, erasing folded velocity and clutter, data thinning, and observation error estimation, are performed. Then, the radar data processed are converted to the model grid for the data assimilation.

\section{c. POD-4DEnVar}

The 4DVar problem can be incrementally formulated as follows (Courtier et al. 1994):

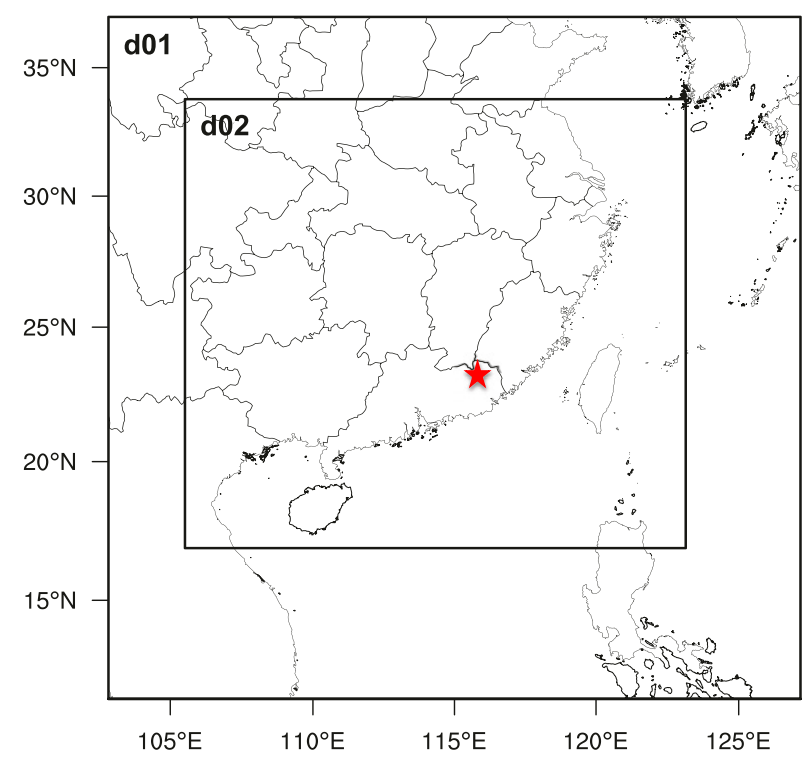

FIG. 2. Spatial coverage of the outer and inner domains (d01 and d02) and the location of the Doppler radar (five-pointed star).

$$
\begin{aligned}
J\left(\mathbf{x}^{\prime}\right)= & \frac{1}{2}\left(\mathbf{x}^{\prime}\right)^{\mathrm{T}} \mathbf{B}^{-1}\left(\mathbf{x}^{\prime}\right)+\frac{1}{2}\left[\mathbf{y}^{\prime}\left(\mathbf{x}^{\prime}\right)\right. \\
& \left.-\left(\mathbf{y}^{\mathrm{obs}}\right)^{\prime}\right]^{\mathrm{T}} \mathbf{R}^{-1}\left[\mathbf{y}^{\prime}\left(\mathbf{x}^{\prime}\right)-\left(\mathbf{y}^{\mathrm{obs}}\right)^{\prime}\right],
\end{aligned}
$$

where $\mathbf{x}^{\prime}=\mathbf{x}-\mathbf{x}_{b}$ is the perturbation of the background field $\mathbf{x}_{b}$ at the analysis time and the matrices $\mathbf{B}$ and $\mathbf{R}$ are the background and observational error covariances, respectively. The observation perturbations $\mathbf{y}^{\prime}\left(\mathbf{x}^{\prime}\right)$ and observation innovations $\left(\mathbf{y}^{\text {obs }}\right)^{\prime}$ are

$$
\begin{aligned}
\mathbf{y}^{\prime}\left(\mathbf{x}^{\prime}\right)=\left[\begin{array}{c}
\mathbf{y}_{1}^{\prime}\left(\mathbf{x}^{\prime}\right) \\
\mathbf{y}_{2}^{\prime}\left(\mathbf{x}^{\prime}\right) \\
\vdots \\
\mathbf{y}_{s}^{\prime}\left(\mathbf{x}^{\prime}\right)
\end{array}\right], \\
\mathbf{y}_{t}^{\prime}\left(\mathbf{x}^{\prime}\right)=\mathbf{y}_{t}^{\prime}\left(\mathbf{x}_{b}+\mathbf{x}^{\prime}\right)-\mathbf{y}_{t}^{\prime}\left(\mathbf{x}_{b}\right), \quad t=1,2, \ldots, s, \\
\left(\mathbf{y}^{\text {obs }}\right)^{\prime}=\left[\begin{array}{c}
\left(\mathbf{y}_{1}^{\text {obs }}\right)^{\prime} \\
\left(\mathbf{y}_{2}^{\text {obs }}\right)^{\prime} \\
\vdots \\
\left(\mathbf{y}_{s}^{\text {obs }}\right)^{\prime}
\end{array}\right], \\
\left(\mathbf{y}_{t}^{\text {obs })^{\prime}}=\mathbf{y}_{t}^{\text {obs }}-\mathbf{y}_{t}\left(\mathbf{x}_{b}\right), \quad t=1,2, \ldots, s,\right.
\end{aligned}
$$

and

$$
\mathbf{y}_{t}(\mathbf{x})=H_{t}\left[M_{1 \rightarrow t}(\mathbf{x})\right]
$$

Here, $t$ is the observation time, $H_{t}$ is the observation operator at time $t, M_{1 \rightarrow t}$ is the forecasting model, and 
$s$ is the total number of observations in the assimilation window.

According to (2) and (3), observation perturbations of $N$ ensemble samples at $t$ times, $\left(\mathbf{y}_{1, t}^{\prime}, \mathbf{y}_{2, t}^{\prime}, \ldots, \mathbf{y}_{N, t}^{\prime}\right)$, $t=1,2, \ldots, s$, denoted by $\mathbf{y}^{p}$ (OPs), are calculated using the observation operator $H_{t}$, the forecasting model $M_{1 \rightarrow t}$, and the model perturbations $\left(\mathbf{x}_{1}^{\prime}, \mathbf{x}_{2}^{\prime}, \ldots, \mathbf{x}_{N}^{\prime}\right)$, which are denoted as $\mathbf{x}^{p}$ (MPs). The POD of the OPs matrix $\mathbf{y}^{p}$ yields

$$
\left(\mathbf{y}^{p}\right)^{\mathrm{T}}\left(\mathbf{y}^{p}\right)=\mathbf{V} \Lambda^{2} \mathbf{V}^{\mathrm{T}},
$$

where the $\mathbf{V}$ matrix is composed of orthogonal vectors and the $\boldsymbol{\Lambda}$ is a diagonal matrix of the corresponding eigenvalues. The POD-transformed OPs, defined as $\mathbf{P}_{y}$, are

$$
\mathbf{P}_{y}=\mathbf{y}^{\prime} \mathbf{V} .
$$

If the assimilation window is no longer than $1 \mathrm{~h}$, assuming a linear relationship between the OPs and the MPs (Wang et al. 2012), we define $\mathbf{P}_{x}$ as the PODtransformed MPs:

$$
\mathbf{P}_{x}=\mathbf{x}^{\prime} \mathbf{V}
$$

The optimal solution $\mathbf{x}^{\prime}$ and its corresponding optimal $\mathbf{y}^{\prime}$ can be expressed by the linear weight combinations of the POD-transformed MPs and OPs, respectively, as follows:

$$
\mathbf{x}^{\prime}=\mathbf{P}_{x} \boldsymbol{\beta},
$$

and

$$
\mathbf{y}^{\prime}=\mathbf{P}_{y} \boldsymbol{\beta},
$$

where $\boldsymbol{\beta}$ is the weight coefficient (Zhang et al. 2015).

Substituting (10) and (11) and the ensemble background covariance $\mathbf{B}=\mathbf{P}_{x} \mathbf{P}_{x}^{\mathrm{T}} /(N-1)$ into (1), and performing some simple calculations, the solution for $\mathbf{x}^{\prime}$ can be expressed as

$$
\mathbf{x}^{\prime}=\mathbf{P}_{x}\left[(N-1) \mathbf{I}+\mathbf{P}_{y}^{\mathrm{T}} \mathbf{R}^{-1} \mathbf{P}_{y}\right]^{-1} \mathbf{P}_{y}^{\mathrm{T}} \mathbf{R}^{-1}\left(\mathbf{y}^{\mathrm{obs}}\right)^{\prime} .
$$

Here, the algorithm assumes the observation errors are additive, unbiased, and Gaussian; $1 \mathrm{~m} \mathrm{~s}^{-1}$ and $1 \mathrm{~dB} Z$ are chosen as the standard deviation of radial velocity and reflectivity observations, respectively.

\section{d. Construction of ensemble samples}

As seen in the above assimilation process, the construction of the ensemble samples is the basis of the implementation of the POD-4DEnVar method. Historical forecasts are employed to generate the ensemble samples in the study, a process referred to as the historical sampling method (Wang et al. 2010).

Figure 3 is a flowchart showing the method, and two sampling runs are conducted with two different sets of initial conditions. For the heavy rainfall event used in this work, two different prediction fields at 1600 UTC 8 December are constructed by model integration from 0000 to 0600 UTC 8 December using FNL data. Then, the two sets of prediction results are used as initial conditions, and two 4-h model integrations from 1600 to 2000 UTC 8 December are conducted, with model output every $6 \mathrm{~min}$, which corresponds to the radar scanning frequency. Since the radar data are assimilated every $6 \mathrm{~min}$, every ensemble sample with model output every $6 \mathrm{~min}$ should also contain 11 time points during the 1-h assimilation window. Thus, 31 ensemble samples can be formed in a 4-h forecast by sliding the time point as follows:

$$
\begin{aligned}
& \left(\mathbf{x}_{1600 \mathrm{UTC}, 08}, \mathbf{x}_{1606 \mathrm{UTC}, 08}, \ldots, \mathbf{x}_{1700 \mathrm{UTC}, 08}\right), \\
& \left(\mathbf{x}_{1606 \mathrm{UTC}, 08}, \mathbf{x}_{1612 \mathrm{UTC}, 08}, \ldots, \mathbf{x}_{1706 \mathrm{UTC}, 08}\right), \\
& \ldots \\
& \left(\mathbf{x}_{1900 \mathrm{UTC}, 08}, \mathbf{x}_{1906 \mathrm{UTC}, 08}, \ldots, \mathbf{x}_{\text {2000UTC }, 08}\right) .
\end{aligned}
$$

So, 62 ensemble samples are collected by using two 4-h forecasts (Fig. 3). Then, the model perturbations $\mathbf{x}^{\prime}$ can be constructed by subtracting the background field $\mathbf{x}_{b}$ from the ensemble samples $\mathbf{x}$.

\section{e. Doppler radar observation operators}

In the radar data assimilation, the radial velocity and the reflectivity are not model variables, so the observation operators are needed when directly assimilating the two sets of observations. The observation operator for radial velocity $V_{r}$ is

$$
V_{r}=u \frac{x-x_{r}}{r}+v \frac{y-y_{r}}{r}+\left(w-V_{\mathrm{Tm}}\right) \frac{z-z_{r}}{r},
$$

where $(u, v, w)$ is the three-dimensional wind field, the variable $r$ is the distance between a model grid point $(x, y, z)$ and the radar location $\left(x_{r}, y_{r}, z_{r}\right)$, and $V_{\mathrm{Tm}}$ is the terminal velocity of the precipitation, which is given by Sun and Crook (1998) as follows:

$$
V_{\mathrm{Tm}}=5.40 a q_{r}^{0.125} \text {. }
$$

The quantity $a$ is a correction factor defined as

$$
a=\left(\frac{P_{0}}{\bar{P}}\right)^{0.4}
$$

in which $\bar{P}$ is the base-state pressure and $P_{0}$ is the pressure at the ground. 


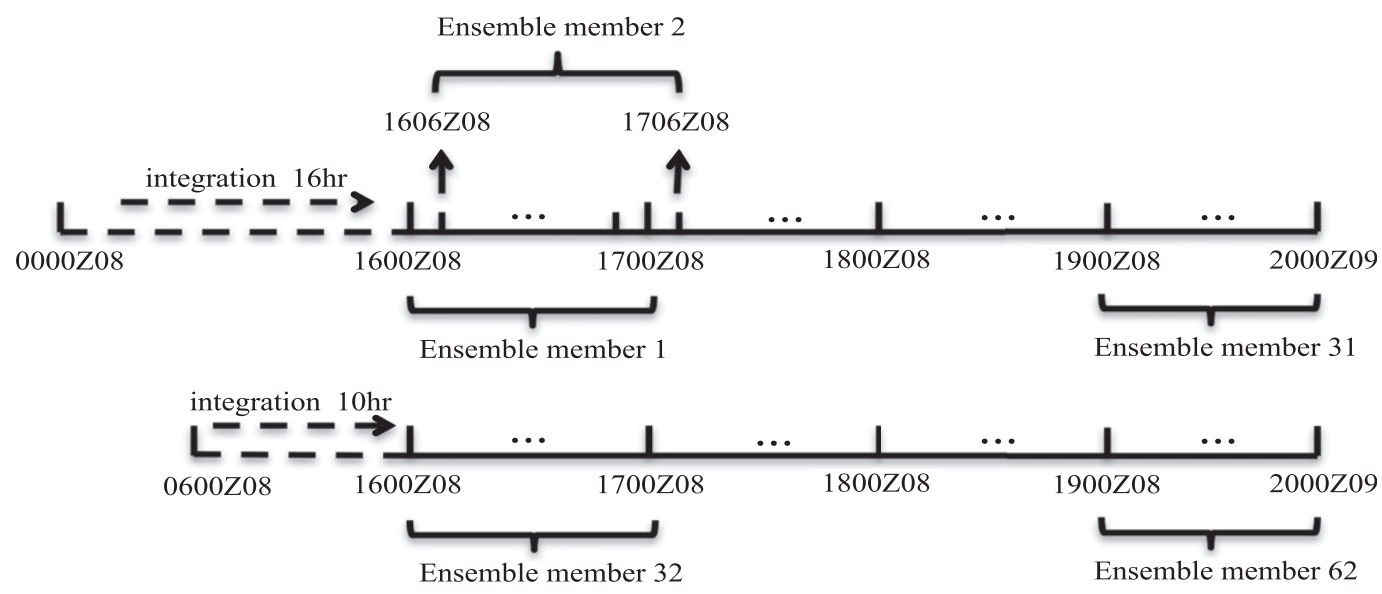

FIG. 3. Flowchart of the historical sampling method.

The observation operator for the reflectivity $Z$ is (Sun and Crook 1997)

$$
Z=43.1+17.5 \log \left(\rho q_{r}\right),
$$

where $\rho$ is the air density and $q_{r}$ is the rainwater mixing ratio.

According to (7), (8), and (12), the results of the data assimilation are related by $\mathbf{P}_{y}$, which is further associated with the orthogonal vectors decomposed by $\mathbf{y}^{p}$. Additionally, it is clear to see from (3) and (13)-(16) that $\mathbf{y}^{p}$ is dependent on the three-dimensional wind field $(u, v, w)$ and the rainwater mixing ratio $q_{r}$. Therefore, the POD-4DEnVar-based Doppler radar data assimilation strongly depends on the accuracy of the threedimensional wind field and the rainwater mixing ratio of the ensemble samples.

\section{Influence of parameterization schemes for the ensemble samples on the POD-4DEnVar data assimilation}

\section{a. Experimental design}

For a complete POD-4DEnVar assimilation experiment, there are three necessary steps. First, using the historical sampling method to construct the ensemble samples. Second, assimilating Doppler radar data and obtaining the analysis field. Third, carrying out the numerical forecast with the analysis field and getting the final result. It is worth noting that choosing different microphysical and cumulus schemes may form different ensemble samples, which will further affect the assimilation and forecast.

Therefore, to explore the influence of the parameterization schemes used for the ensemble samples on the POD-4DEnVar data assimilation, 16 sensitivity tests based on four microphysics schemes and four cumulus schemes in the WRF Model are designed. Each test constructs 62 ensemble samples with the historical sampling method. In addition, an experiment without radar data assimilation (referred to as CNTL) is conducted to evaluate the effect of the different parameterization schemes. The CNTL run is initialized using the reanalysis data (the first-guess field) at 1900 UTC 8 December 2015. To exclude the other factors, the 16 sensitivity tests and the CNTL experiment all adopt the same parameterization schemes listed in section $2 \mathrm{~b}$ during the forecast stage (Fig. 4, Table 1).

\section{b. Results}

To visualize the effect of the various parameterization schemes chosen to construct the ensemble samples, Fig. 5 shows 24 -h accumulated precipitation simulated by the 16 sensitivity tests. Compared with the observed precipitation (Fig. 1), it can be seen that although these sensitivity tests generally capture the location of the rainband, the intensity of the precipitation varies greatly among the different tests (Fig. 5). Experiments mp1_cu3, mp2_cu5, and mp6_cu3 (Figs. 5c,h,o) successfully simulate the rainstorm center in the east of Guangdong Province and the south of Fujian Province, and their results are the closest to the observed precipitation. The precipitation intensities of experiments mp1_cu1, mp1_cu2, mp1_cu5, mp2_cu2, mp6_cu2, and mp6_cu5 (Figs. 5a,b,d,f,n,p) in the rainstorm area are much stronger than the observed precipitation, while the precipitation intensities of experiments mp2_cu1, mp2_cu3, mp3_cu1, mp3_cu2, mp3_cu3, mp3_cu5, and mp6_cu1 (Figs. 5e,g, i, j,k,l,m) are much weaker, and this last group of experiments fails to simulate the rainstormlevel precipitation in Guangdong and Fujian Provinces. In summary, it can be seen that the choice of 


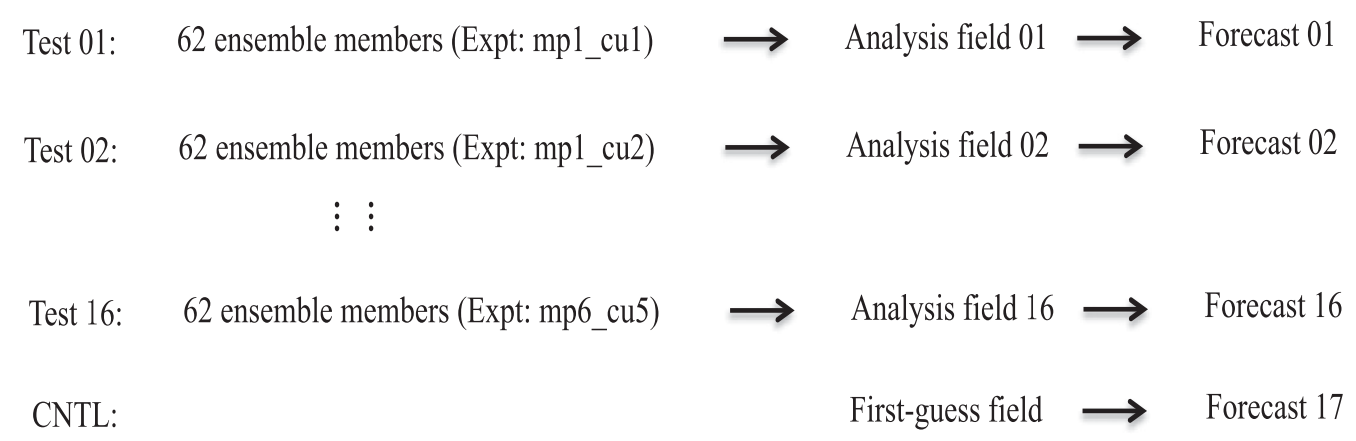

FIG. 4. Flowchart of the 16 sensitivity tests and the CNTL experiment.

parameterization scheme greatly influences the numerical prediction results when constructing ensemble samples using the historical sampling method.

In an attempt to identify the reasons for the differences in precipitation among the various sensitivity experiments, the hourly precipitation deviation evolution of each experiment is further analyzed (Fig. 6) using the prediction results of the CNTL experiment as the reference field. As seen in Figs. 6a,c,e,g, when the cumulus scheme is fixed, different microphysics schemes will result in significantly different simulated precipitation. The hourly precipitation deviation obtained by the WSM3 scheme (blue line) is the largest, followed by the Lin scheme (red line), the WSM6 scheme (green line), and the Kessler scheme (black line). Similarly, Figs. 6b,d,f,h show that when the microphysics scheme is constant, the choice of cumulus scheme also has a significant impact on the precipitation forecast. Choosing the BettsMiller-Janjić (red line) or Grell-3 (green line) scheme as the cumulus scheme results in smaller deviations of hourly precipitation from the CNTL experiment than the Kain-Fritsch or Grell-Freitas schemes.

In general, it can be seen that the parameterization scheme of the ensemble samples plays an important role in the numerical forecast. The cumulus and microphysics schemes primarily affect forecast precipitation. Therefore, the influence of the parameterization schemes used for the ensemble samples should be considered when assimilating radar data using the POD-4DEnVar method.

\section{c. Analysis}

In the sensitivity tests of the parameterization schemes described above, only the schemes of the ensemble samples are adjusted, but the schemes of the forecast stage remain the same for all the tests. Therefore, the differences in the precipitation among the tests are not related to the forecast stage but are related to the differences in the analysis fields. Figure 7 shows the changes in wind field and water vapor mixing ratio at $850 \mathrm{hPa}$ relative to the CNTL experiment at $1900 \mathrm{UTC}$ 8 December 2015 for each sensitivity test. It is clear that 16 tests have different increments relative to the CNTL experiment in these two variables. The reason is that the different parameterization schemes result in different ensemble samples, and lead to different assimilation analysis fields. So the ensemble samples are very important in the radar data assimilation using POD-4DEnVar with the physical ensemble method.

No apparent differences in changes to the vertical velocity relative to the CNTL experiment at the analysis time were found among the 16 sensitivity tests (not shown). However, further analysis shows that the vertical

TABLE 1. List of the sensitivity tests and their different parameterization schemes. Note that the CNTL experiment without radar data assimilation is conducted to be the reference test.

\begin{tabular}{clll}
\hline \hline & & $\begin{array}{c}\text { Microphysics } \\
\text { scheme }\end{array}$ & Cumulus scheme \\
\hline 01 & mp1_cu1 & Kessler & Kain-Fritsch \\
02 & mp1_cu2 & Kessler & Betts-Miller-Janjić \\
03 & mp1_cu3 & Kessler & Grell-Freitas \\
04 & mp1_cu5 & Kessler & Grell-3 \\
05 & mp2_cu1 & Lin & Kain-Fritsch \\
06 & mp2_cu2 & Lin & Betts-Miller-Janjić \\
07 & mp2_cu3 & Lin & Grell-Freitas \\
08 & mp2_cu5 & Lin & Grell-3 \\
09 & mp3_cu1 & WSM3 & Kain-Fritsch \\
10 & mp3_cu2 & WSM3 & Betts-Miller-Janjić \\
11 & mp3_cu3 & WSM3 & Grell-Freitas \\
12 & mp3_cu5 & WSM3 & Grell-3 \\
13 & mp6_cu1 & WSM6 & Kain-Fritsch \\
14 & mp6_cu2 & WSM6 & Betts-Miller-Janjić \\
15 & mp6_cu3 & WSM6 & Grell-Freitas \\
16 & mp6_cu5 & WSM6 & Grell-3 \\
\hline
\end{tabular}



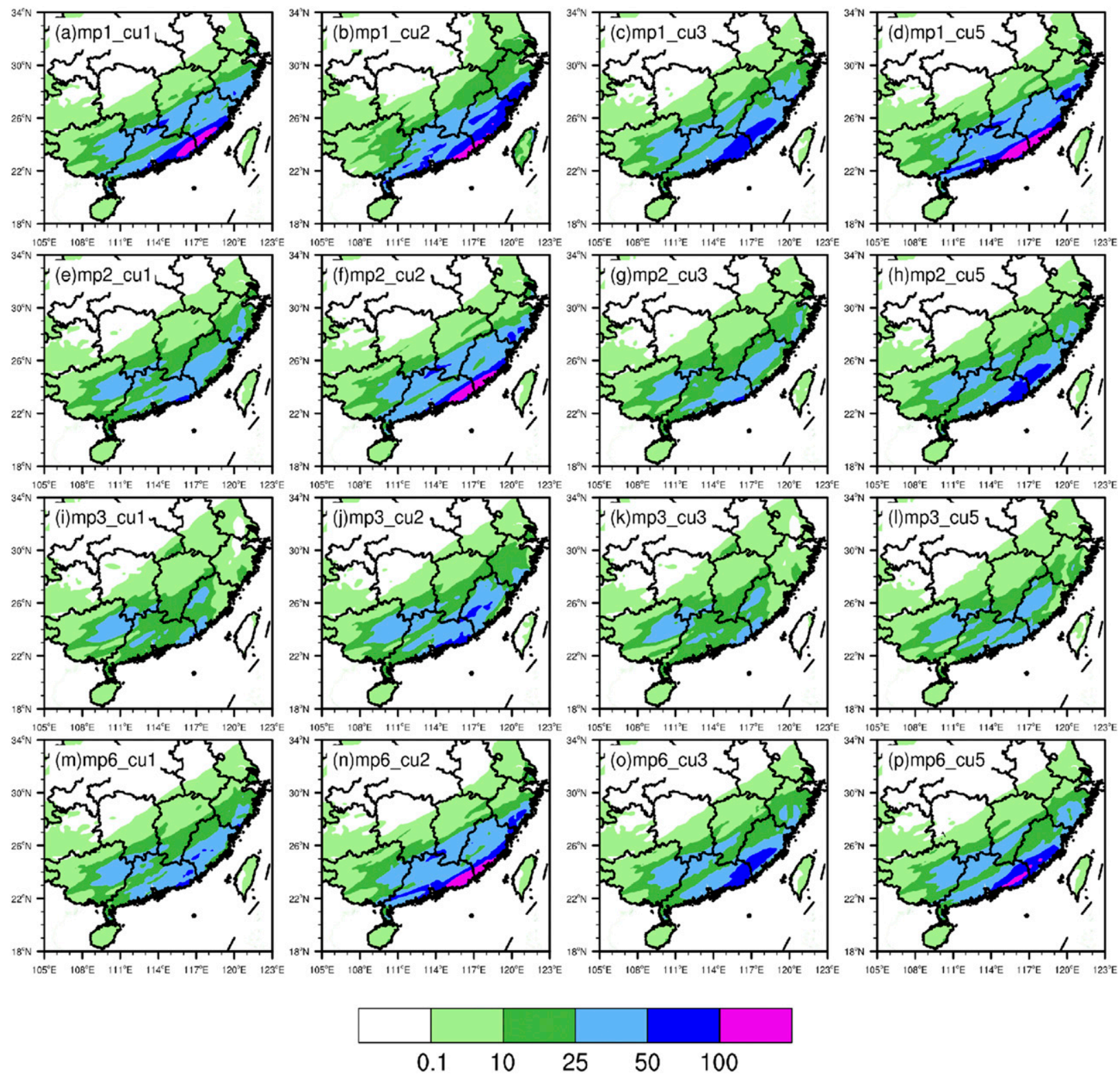

FIG. 5. The 24-h accumulated precipitation (mm) from 2000 UTC 8 Dec to 2000 UTC 9 Dec 2015 for 16 sensitivity tests.

velocity increment of the precipitation center (along $116.5^{\circ} \mathrm{E}$ ) in each test increases significantly after two model integration hours (Fig. 8). The vertical velocity responds differently in the various sensitivity tests, and the adjustment to vertical velocity is correlated with that of the water vapor mixing ratio. That is, tests with large changes to the water vapor mixing ratio also have large changes in vertical velocity.

It is found that changes in water vapor mixing ratio and vertical velocity among the 16 sensitivity tests (Fig. 8) result in changes in hourly precipitation (Fig. 6). Specifically, when the water vapor mixing ratio and vertical velocity are reduced, the hourly precipitation does decrease. The change in hourly precipitation will also be small when the water vapor mixing ratio and the vertical velocity are not changed significantly. Consequently, if the parameterization scheme used for the ensemble samples changes, the analysis field obtained from assimilating radar data using the POD-4DEnVar method will also change, thus affecting the precipitation forecast.

The above analysis shows that radar data assimilation by POD-4DEnVar is sensitive to the parameterization schemes used for the ensemble samples, and different 

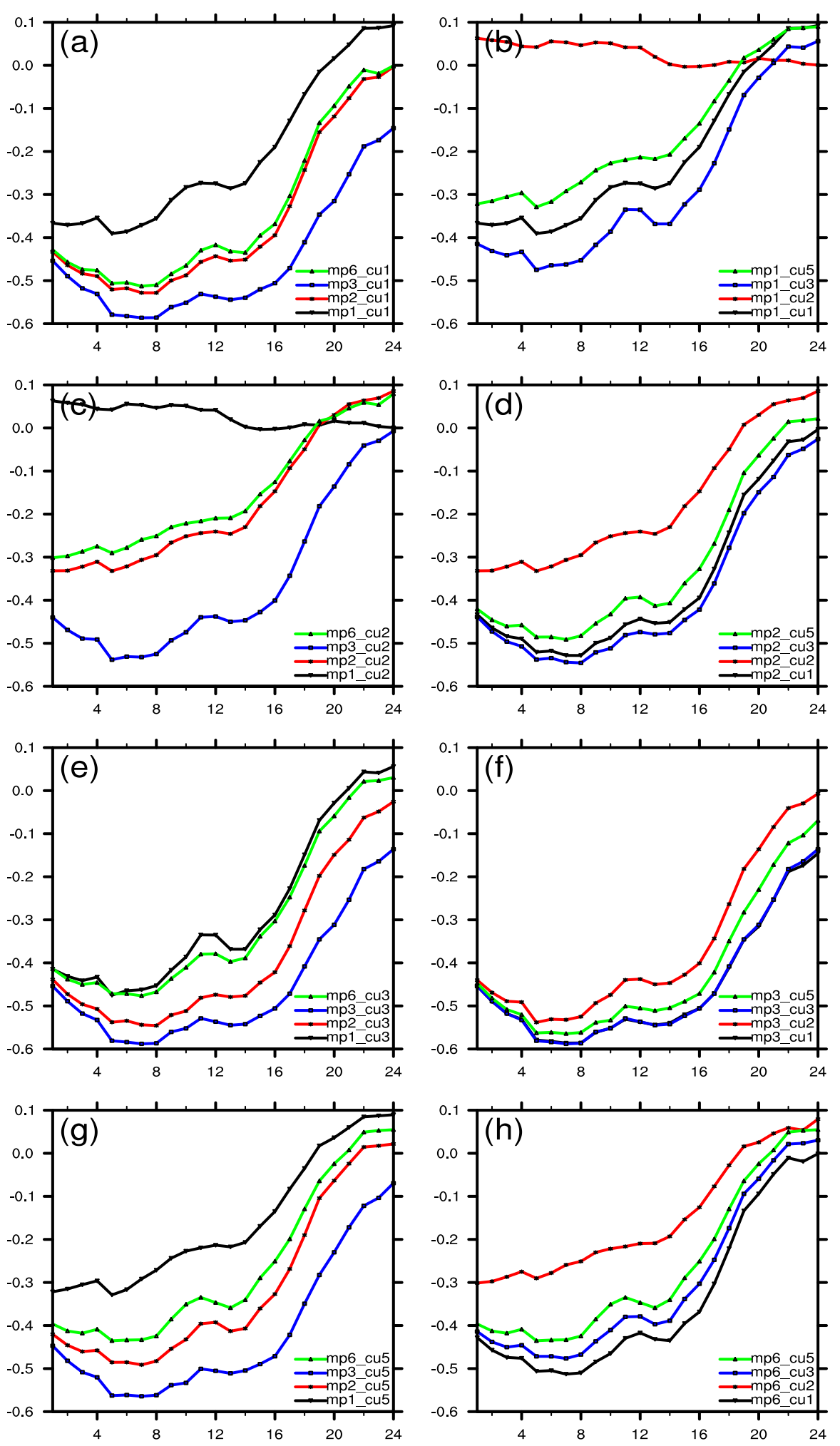

FIG. 6. Evolution of the hourly precipitation deviation $(\mathrm{mm})$ for the sensitivity tests with different (a),(c),(e),(g) microphysics and (b),(d),(f),(h) cumulus schemes. 

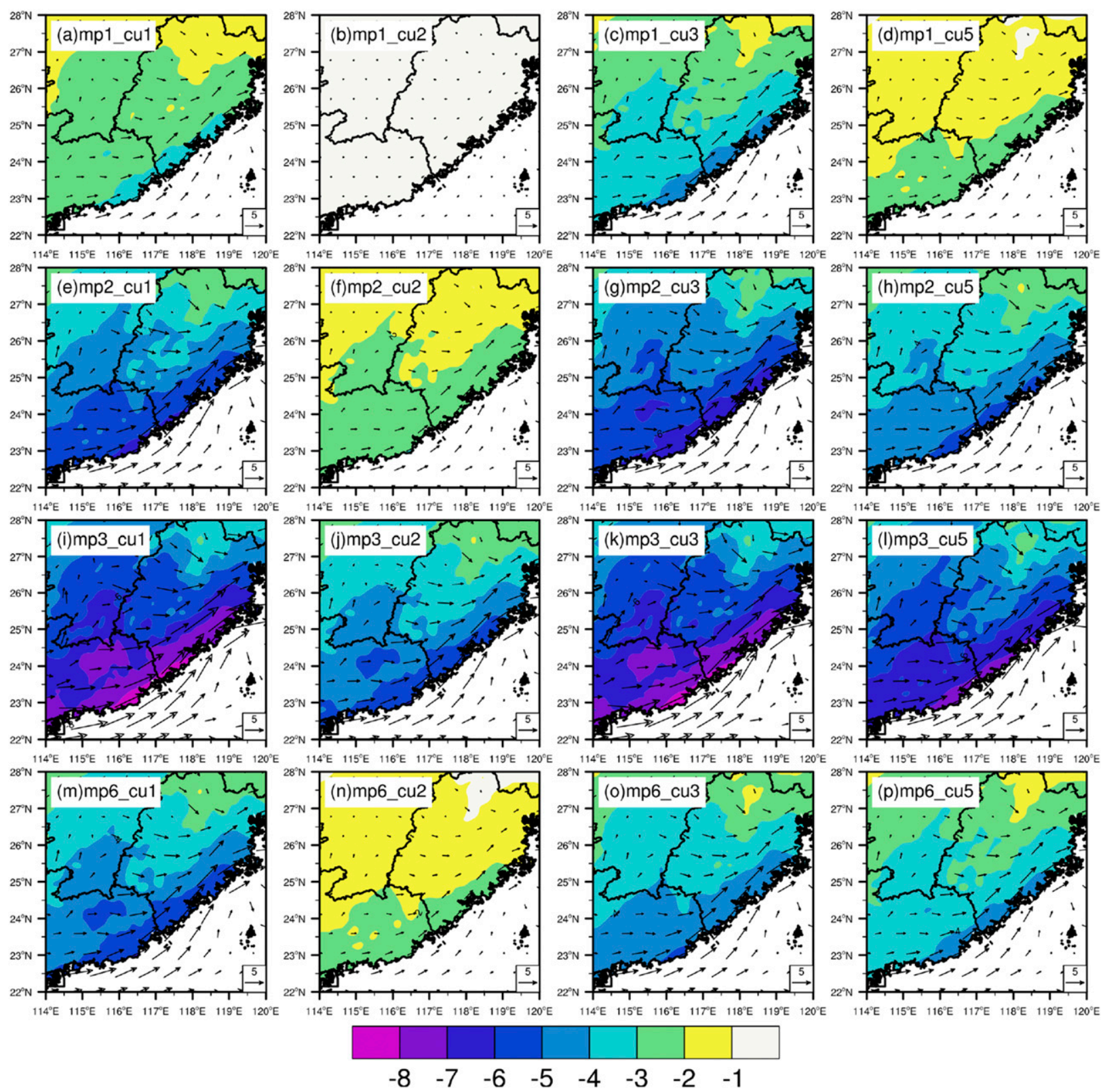

FIG. 7. The increments for wind field (vectors; $\mathrm{m} \mathrm{s}^{-1}$ ) and water vapor mixing ratio (shaded; $\mathrm{g} \mathrm{kg}^{-1}$ ) at $850 \mathrm{hPa}$ for 16 sensitivity tests at 1900 UTC 8 Dec 2015 relative to the CNTL experiment.

schemes will lead to different analysis fields, which are in turn the direct cause of the prediction differences in precipitation among the 16 sensitivity tests. To further explore how the parameterization scheme affects the assimilation, the spread of the wind and water vapor fields of the ensemble samples with different schemes are compared.

Small spread among the ensemble samples of the 16 sensitivity tests were found for the horizontal wind field (not shown), which means that different parameterization schemes have little influence on the horizontal wind field of the ensemble samples. Figure 9 shows the spread of the vertical velocity and the rainwater mixing ratio at the analysis time for the ensemble samples of the 16 sensitivity tests. It can be seen from Fig. 9 that both cumulus and microphysical schemes have effects on the vertical velocity (Figs. 9a-d), but the rainwater mixing ratio is more sensitive to microphysical processes than to cumulus convection processes (Figs. 9e-h). This shows that the ensemble samples with different physical schemes will lead to distinctly different vertical velocity and rainwater mixing ratios at the analysis time. So the 

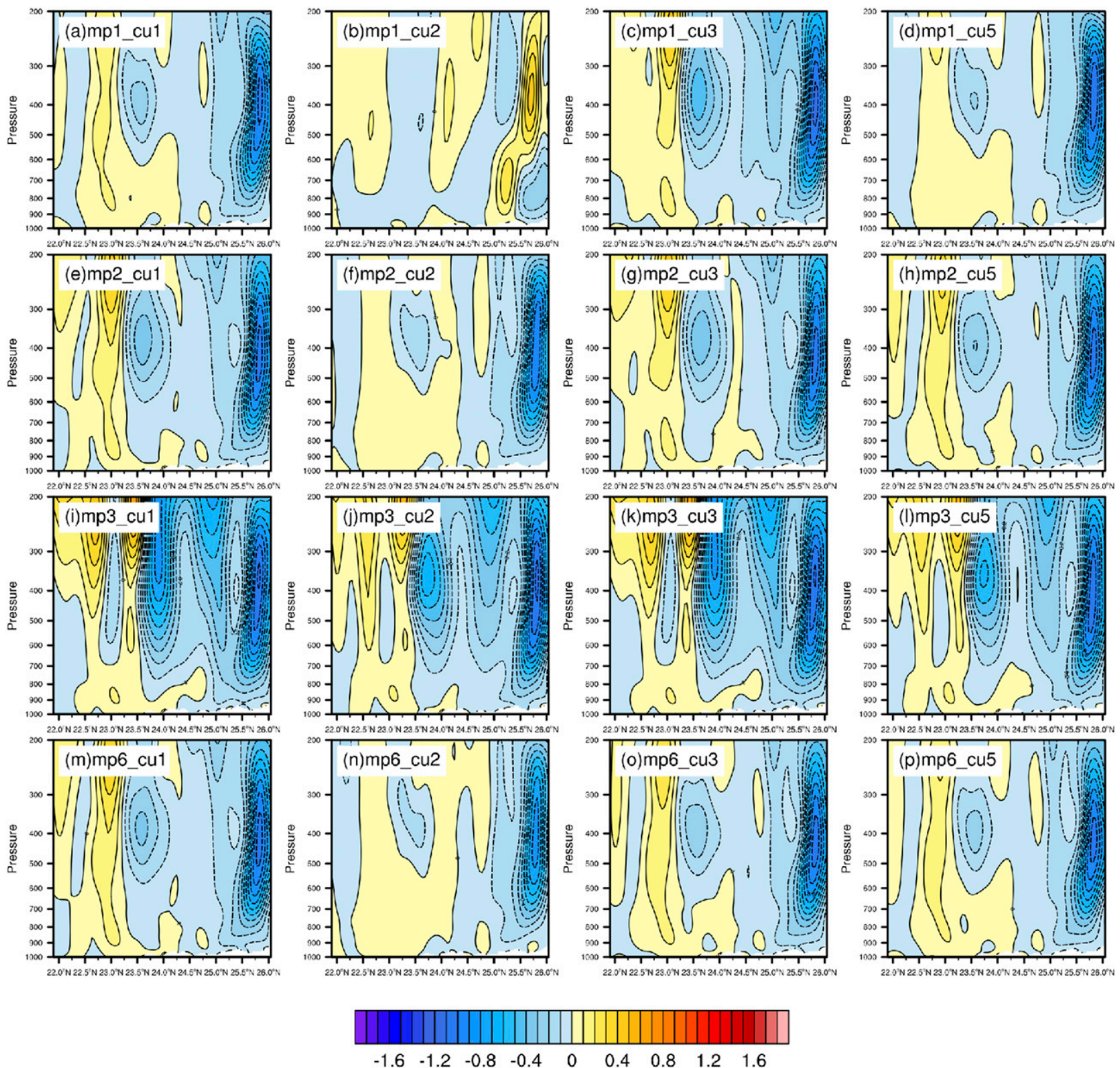

FIG. 8. North-south vertical cross section of changes to vertical velocity $\left(\mathrm{m} \mathrm{s}^{-1}\right)$ along $116.5^{\circ} \mathrm{E}$ for 16 sensitivity tests at $2100 \mathrm{UTC} 8 \mathrm{Dec}$ 2015 relative to the CNTL experiment.

radar data assimilation by the POD-4DEnVar based on these ensemble samples must lead to different prediction results.

From the analysis above, it is evident that adopting different cumulus and microphysics schemes will lead to different simulations of the vertical velocity and rainwater mixing ratio. Jankov et al.'s (2005) and Cossu and Hocke's (2014) research also supports this conclusion. These differences in the ensemble samples are introduced into the POD-4DEnVar assimilation by the radar data observation operator, which may further cause the differences in precipitation forecasts of the sensitivity tests.

\section{EVA based on physical ensemble}

\section{a. Experimental design}

The sensitivity tests in section 3 clearly show that the vertical velocity and rainwater mixing ratio will differ with the choice of parameterization scheme used to construct the ensemble samples and contribute to differences in the corresponding assimilation and prediction results. To improve the accuracy of the assimilation and prediction results, the physical ensemble technique, which can weaken the influence of the parameterization schemes (Meng and Zhang 2007; Lan et al. 2010) 

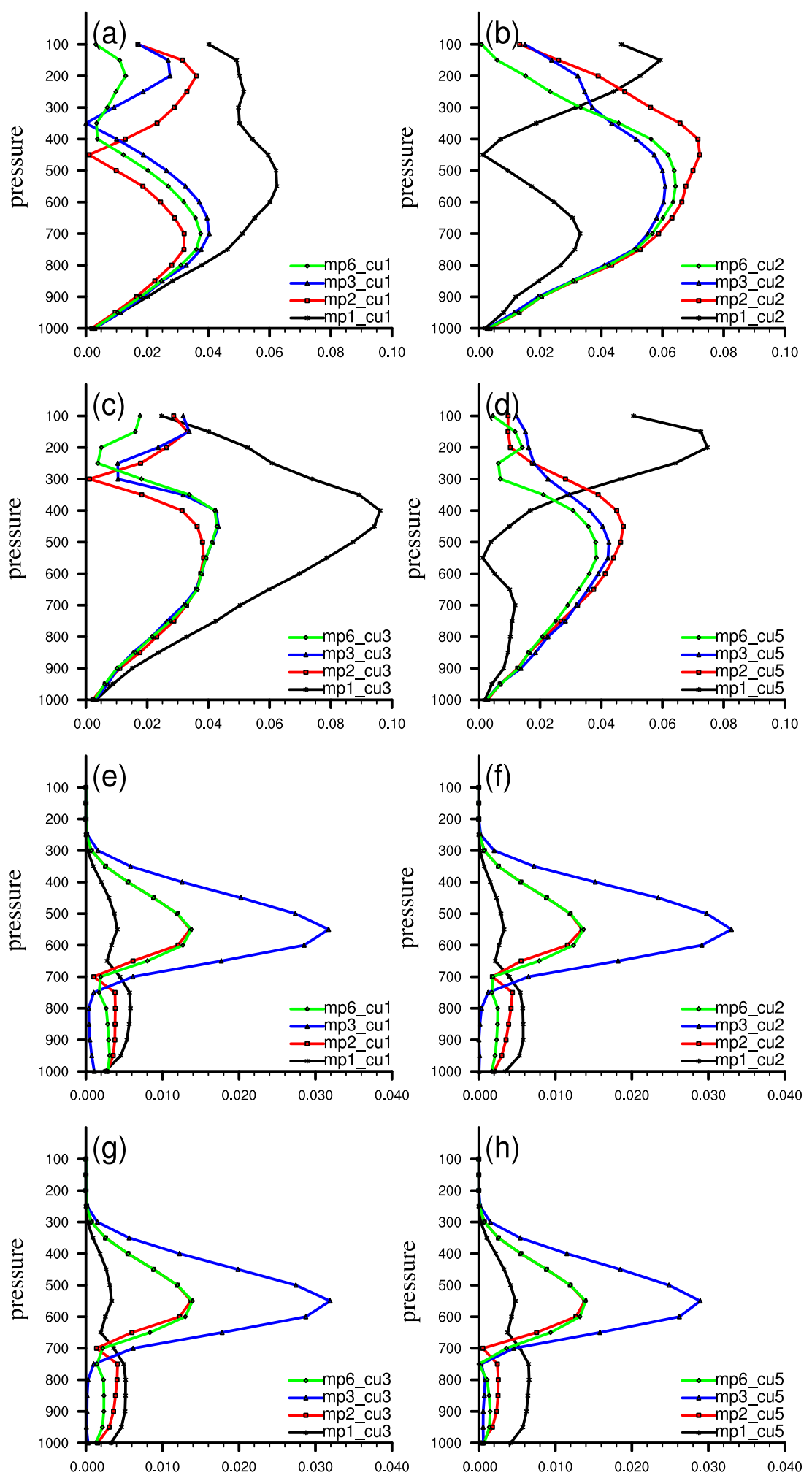

FIG. 9. The spread of (a)-(d) vertical velocity $\left(10^{-2} \mathrm{~m} \mathrm{~s}^{-1}\right)$ and (e)-(h) rainwater mixing ratio $\left(\mathrm{g} \mathrm{kg}^{-1}\right)$ in the vertical for the sensitivity tests with different microphysics schemes and cumulus schemes at 1900 UTC 8 Dec 2015. 
TABLE 2. Descriptions of the three assimilation experiments based on the physical ensemble technique.

\begin{tabular}{ccc}
\hline Expt & Name & Description \\
\hline 1 & Multiphy-assimilation & $\begin{array}{c}\text { Ensemble samples are constructed using the 16 combinations of schemes listed in } \\
\text { Table 1; the forecasting model uses a fixed parameterization scheme } \\
\text { The } 16 \text { tests shown in Table } 1 \text { are carried out and the ensemble average of the } 16 \\
\text { analysis fields is used as the initial field for a numerical forecast; the forecasting } \\
\text { model is the same as in the first experiment }\end{array}$ \\
& The 16 tests shown in Table 1 are carried out and the 16 analysis fields are used for \\
& Multiphy-forecast & numerical forecasts; the ensemble average of the 16 numerical forecasts is \\
& used as the result; the forecasting model is the same as in the first experiment
\end{tabular}

is used to develop three experiments (Table 2). For the first experiment (Multiphy-assimilation), ensemble samples are constructed using 16 tests with the different parameterization schemes listed in Table 1 and each test also contains 62 ensemble samples with the historical sampling method. As a result, a total of $992(16 \times 62)$ ensemble samples constructed are used for data assimilation. Then, a numerical forecast is conducted with the analysis field. For the second experiment (Multiphyanalysis), the 16 tests shown in Table 1 are carried out and the ensemble average of the corresponding 16 analysis fields are used as the initial field for a numerical forecast. For the third experiment (Multiphy-forecast), the assimilation is the same as that of Multiphy-analysis. However, the 16 analysis fields are used for the numerical forecast, and the ensemble average of the $16 \mathrm{nu}-$ merical forecasts is used as the forecast result for Multiphy-forecast. The specific processes used in the three experiments are illustrated in Fig. 10.

To quantitatively assess the analysis fields of the three experiments, a "true" atmospheric state for the analysis field is required. Similar to Houtekamer and Mitchell (2005), a random perturbation, which is derived from the background error covariance of the WRF 3DVar data assimilation system, is added to the background field at 1900 UTC 7 December 2015. Then, an assumed true run is integrated for $24 \mathrm{~h}$ with the perturbed background field. Thus, the simulated true atmospheric state at 1900 UTC 08 December 2015 is generated. The CNTL experiment without radar data assimilation is still used as the reference experiment, as described above.

\section{b. Results}

The 24-h accumulated precipitation from 2000 UTC 8 December to 2000 UTC 9 December 2015 for the CNTL experiment, as well as the simulated precipitation from the three assimilation experiments, are shown in Fig. 11. Comparing these results with the observations (Fig. 1a), all four experiments capture the general distribution of the rain belt, but there are obvious differences in the simulated precipitation intensity.
The CNTL experiment predicts the heaviest rainfall among the four experiments, with a maximum precipitation intensity exceeding $100 \mathrm{~mm}$ (Fig. 11a). In contrast, the precipitation intensity of the three assimilation experiments is significantly reduced. The Multiphyassimilation experiment almost fails to predict the heavy rainfall that occurred in eastern Guangdong and southern Fujian Provinces (Fig. 11b), while Multiphyanalysis (Fig. 11c) and Multiphy-forecast (Fig. 11d) both simulate precipitation that is very close to the observations. Comparing the Multiphy-analysis and Multiphyforecast results, it can be seen that the difference between the two experiments lies primarily in the precipitation in southern Fujian Province. The location of the rainstorm (above 50-mm precipitation) is shifted slightly north for Multiphy-analysis, while the scope of the precipitation is a slightly smaller than that observed for Multiphy-forecast.

To quantitatively evaluate the precipitation prediction, the structure $S$, amplitude $A$, and location $L$ quantitative assessment method (SAL) proposed by Wernli et al. (2008) is adopted. The smaller the absolute values of $S, A$, and $L$ are, the closer the precipitation forecast is to the observations, which means better precipitation forecasts. Figure 12 shows the SAL scores for the CNTL and three assimilation experiments relative to the observations.

The values of $L$ for Multiphy-analysis and Multiphyforecast are slightly less than those of CNTL and Multiphy-assimilation, which indicates that the forecasts of the location for Multiphy-analysis and Multiphyforecast are better than those of the other two experiments. Also, the absolute value of $A$ follows the trend of $L$ for these four experiments, which indicates that the intensity of the precipitation simulated by Multiphyanalysis and Multiphy-forecast is also better than that of CNTL and Multiphy-assimilation. Comparing the absolute value of $S$ among the four experiments, we find that CNTL has the worst precipitation structure prediction, followed by Multiphy-assimilation and Multiphyanalysis. The Multiphy-forecast simulation has the best precipitation structure prediction. 


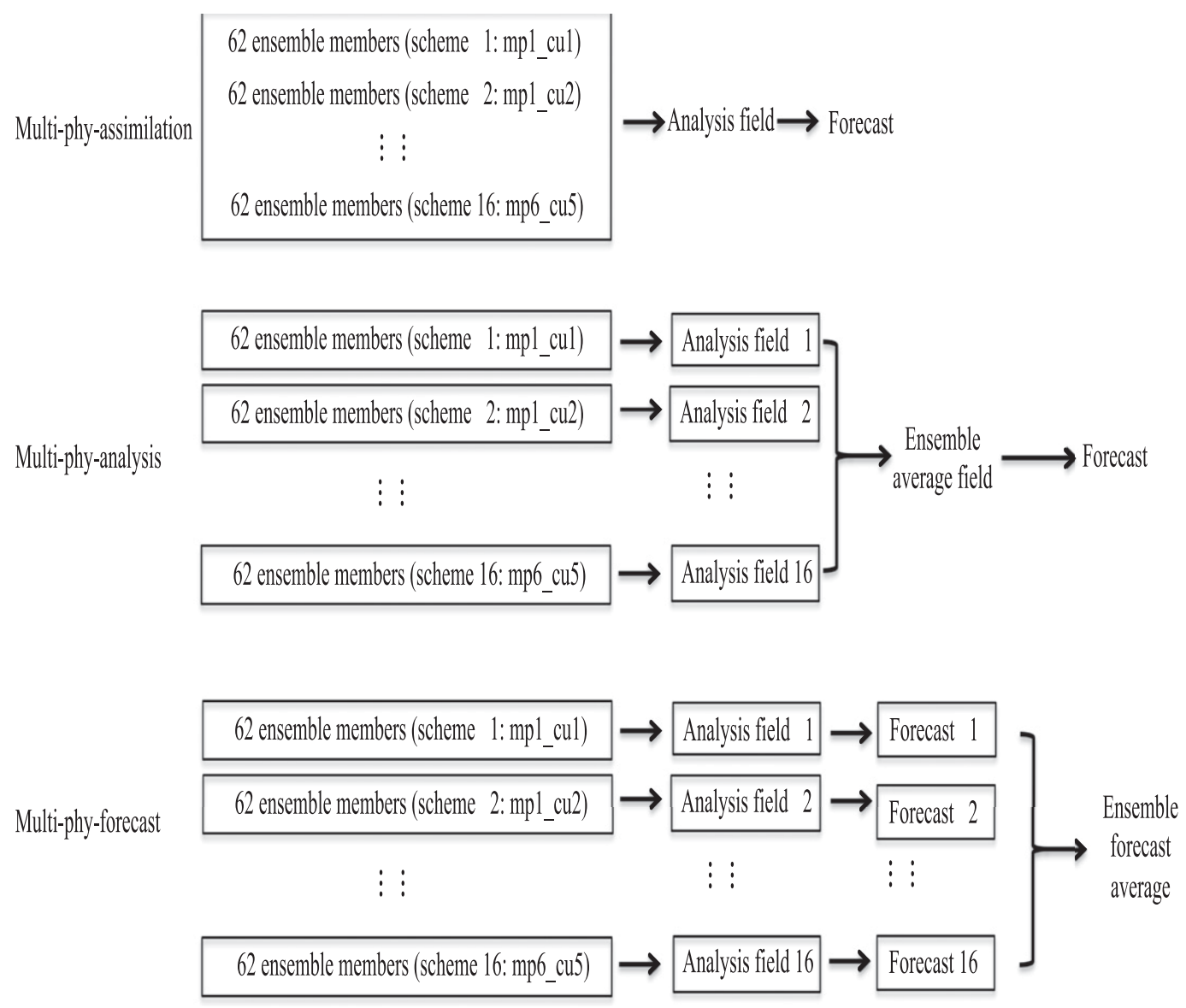

FIG. 10. Flowchart of data assimilation based on the physical ensemble technique.

Based on the above analysis, it can be seen that the precipitation forecasts are significantly improved by considering the influence of the parameterization schemes compared with the CNTL experiment. Additionally, the simulated precipitation of Multiphyforecast is better than that of the other two assimilation experiments.

\section{c. Analysis}

In section $4 \mathrm{~b}$, we demonstrated that the precipitation predictions of the experiments based on the physical ensemble technique are more accurate than those of the CNTL experiment, but different physical ensemble methods lead to varying degrees of improvement. Among the three assimilation experiments, Multiphy-assimilation takes the approach of the physical ensemble before assimilation, Multiphyanalysis averages the initial fields of the tests with different parameterization schemes after assimilation, and Multiphy-forecast averages the numerical forecasts of multiple tests after the numerical predictions (Fig. 10).
The Multiphy-assimilation and Multiphy-analysis simulations are analyzed to explore the reason why the forecasted precipitation results of the two experiments are different. The domain-averaged vertical profiles of the root-mean-square errors (RMSEs) of different variables from Multiphy-assimilation and Multiphy-analysis at 1900 UTC 8 December 2015 are shown in Fig. 13. It is clear that the RMSEs of the variables in Multiphy-analysis are smaller than those in Multiphy-assimilation, which indicates that the analysis field obtained by Multiphy-analysis is closer to the trueatmospheric state than Multiphy-assimilation. Therefore, the slight difference in the initial field caused by different physical ensemble methods is the reason for the difference in the simulated precipitation between Multiphy-assimilation and Multiphy-analysis.

Unlike the difference in precipitation between Multiphy-assimilation and Multiphy-analysis, it is the numerical forecast after assimilation that leads to the different precipitation predictions between Multiphy-analysis and Multiphy-forecast. For the numerical forecast of the two experiments, different 

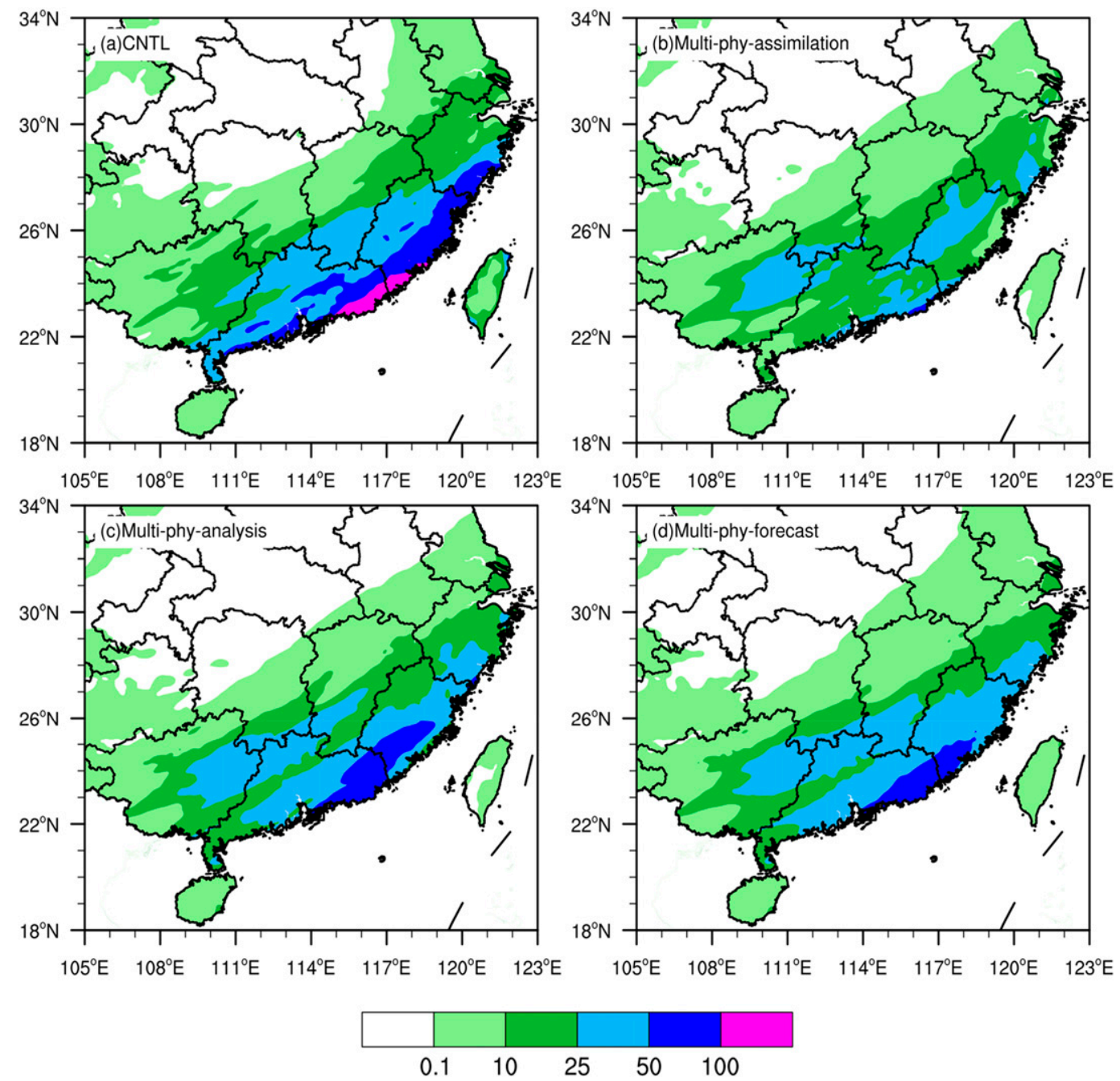

FIG. 11. The 24-h accumulated precipitation (mm) from 2000 UTC 8 Dec to 2000 UTC 9 Dec 2015 for (a) CNTL,

(b) Multiphy-assimilation, (c) Multiphy-analysis, and (d) Multiphy-forecast.

physical ensemble methods are adopted, which can be expressed as

$$
\mathbf{X}_{2}^{f}=M\left(\frac{1}{n} \sum_{i=1}^{n} \mathbf{X}_{i}^{a}\right)
$$

and

$$
\mathbf{X}_{3}^{f}=\frac{1}{n} \sum_{i=1}^{n} M\left(\mathbf{X}_{i}^{a}\right)
$$

where $\mathbf{X}_{i}^{a}$ is the $i$ th analysis field; $\mathbf{X}_{2}^{f}$ and $\mathbf{X}_{3}^{f}$ represent the forecast fields of Multiphy-analysis and Multiphyforecast, respectively; $M$ is the nonlinear forecasting model; and $n$ is the number of analysis fields, which is 16 in this study.

The ensemble average of $n$ analysis fields is denoted by $\overline{\mathbf{X}}^{a}$, and each analysis field is considered to be a corresponding small perturbation (referred to as $\delta \mathbf{X}_{i}^{a}$ ) superimposed on the ensemble average $\overline{\mathbf{X}}^{a}$, which can be defined as

$$
\overline{\mathbf{X}}_{i}^{a}=\overline{\mathbf{X}}^{a}+\delta \mathbf{X}_{i}^{a}(i=1,2, \ldots, n)
$$

Substituting (19) into (17) and (18), the two forecast fields can be expressed as

$$
\mathbf{X}_{2}^{f}=M\left[\frac{1}{n} \sum_{i=1}^{n}\left(\overline{\mathbf{X}}^{a}+\delta \mathbf{X}_{i}^{a}\right)\right]
$$

and

$$
\mathbf{X}_{3}^{f}=\frac{1}{n} \sum_{i=1}^{n} M\left(\overline{\mathbf{X}}^{a}+\delta \mathbf{X}_{i}^{a}\right)
$$




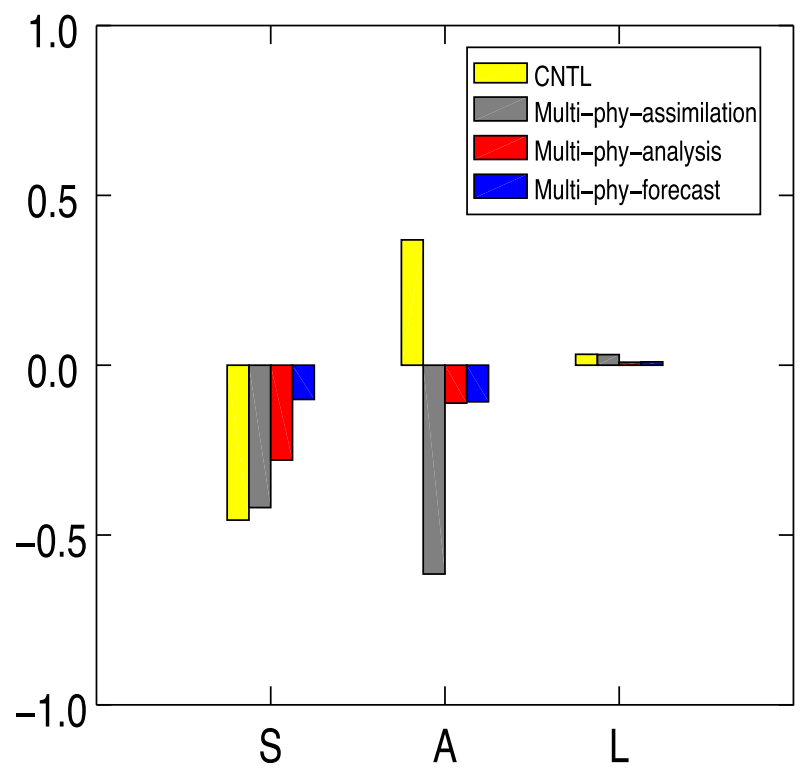

FIG. 12. The SAL scores of 24-h accumulated precipitation for the CNTL, Multiphy-assimilation, Multiphy-analysis, and Multiphy-forecast experiments.

After a Taylor expansion of (20) and (21), the two equations can be written as follows:

$$
\begin{aligned}
\mathbf{X}_{2}^{f}= & M\left(\overline{\mathbf{X}}^{a}\right)+\frac{\partial M}{\partial \mathbf{X}}\left(\frac{1}{n} \sum_{i=1}^{n} \delta \mathbf{X}_{i}^{a}\right)+\frac{1}{2 !} \frac{\partial^{2} M}{\partial \mathbf{X}^{2}}\left(\frac{1}{n} \sum_{i=1}^{n} \delta \mathbf{X}_{i}^{a}\right)^{2} \\
& +\cdots+\frac{1}{n !} \frac{\partial^{n} M}{\partial \mathbf{X}^{n}}\left(\frac{1}{n} \sum_{i=1}^{n} \delta \mathbf{X}_{i}^{a}\right)^{n}
\end{aligned}
$$

and

$$
\begin{aligned}
\mathbf{X}_{3}^{f}= & M\left(\overline{\mathbf{X}}^{a}\right)+\frac{\partial M}{\partial \mathbf{X}}\left(\frac{1}{n} \sum_{i=1}^{n} \delta \mathbf{X}_{i}^{a}\right)+\frac{1}{2 !} \frac{\partial^{2} M}{\partial \mathbf{X}^{2}}\left[\frac{1}{n} \sum_{i=1}^{n}\left(\delta \mathbf{X}_{i}^{a}\right)^{2}\right] \\
& +\cdots+\frac{1}{n !} \frac{\partial^{n} M}{\partial \mathbf{X}^{n}}\left[\frac{1}{n} \sum_{i=1}^{n}\left(\delta \mathbf{X}_{i}^{a}\right)^{n}\right]
\end{aligned}
$$

Here, (22) and (23) represent the numerical processes of Multiphy-analysis and Multiphy-forecast, respectively. Because the forecasting model $M$ is nonlinear, the forecasts of the two experiments are expected to be different. Also, it is evident that although the initial fields of Multiphy-analysis contain the uncertainty introduced by different parameterization schemes, this uncertainty has been filtered out by the ensemble average of the analysis fields before the numerical forecast, which is represented by the term $(1 / n) \sum_{i=1}^{n} \delta \mathbf{X}_{i}^{a}$ in (22). In contrast, Multiphy-forecast performs the numerical forecasts with different analysis fields and averages the forecasts of multiple tests, which allows the small deviations of the analysis fields to be retained when performing the numerical forecast. Consequently, the uncertainty brought by different parameterization schemes is ultimately reflected in the forecast results of the numerical model and is represented by the term $(1 / n) \sum_{i=1}^{n}\left(\delta \mathbf{X}_{i}^{a}\right)^{p}(p=1,2, \ldots, n)$ in (23). In fact, Multiphy-forecast is the ensemble forecast but with the initial fields of the ensemble forecast originating from the assimilation results with different parameterization schemes. Results from Multiphy-forecast include not only the influence of the parameterization schemes on the assimilation but also chain effects brought by the initial field difference on the numerical forecast.

In summary, the uncertainty of the initial fields still exists even if the ensemble variational assimilation method is used at the analysis time because of the different physical processes in the model, but utilizing an appropriate physical ensemble technique during the forecast period can be an effective means of reducing this uncertainty, thus leading to a better numerical forecast.

\section{Conclusions}

Using the WRF Model and Doppler radar data, this study carried out four-dimensional ensemble variational assimilation experiments based on POD-4DEnVar and discussed the sensitivity of the POD-4DEnVar-based radar data assimilation to the parameterization schemes of the ensemble samples. To obtain the optimal precipitation prediction, three methods based on the physical ensemble technique are proposed. The main conclusions are as follows.

1) The parameterization schemes of the numerical model have a significant influence on the simulated precipitation. For the heavy rainfall event used in this study, cumulus schemes and microphysics schemes both affect the simulated precipitation intensity. Therefore, the influence of the parameterization schemes in the ensemble samples must be considered when assimilating radar data using POD-4DEnVar.

2) For the POD-4DEnVar-based radar data assimilation, selecting different parameterization schemes to form ensemble samples leads to different analysis fields, and these differences are the direct cause of the different precipitation prediction results. Compared with the CNTL experiment, the hourly precipitation decreases when the water vapor and the vertical velocity are reduced but remains relatively constant when water vapor and vertical velocity are not significantly changed.

3) Cumulus and microphysics schemes primarily affect the vertical velocity and rainwater mixing ratio of the ensemble forecasts. These effects are introduced into 

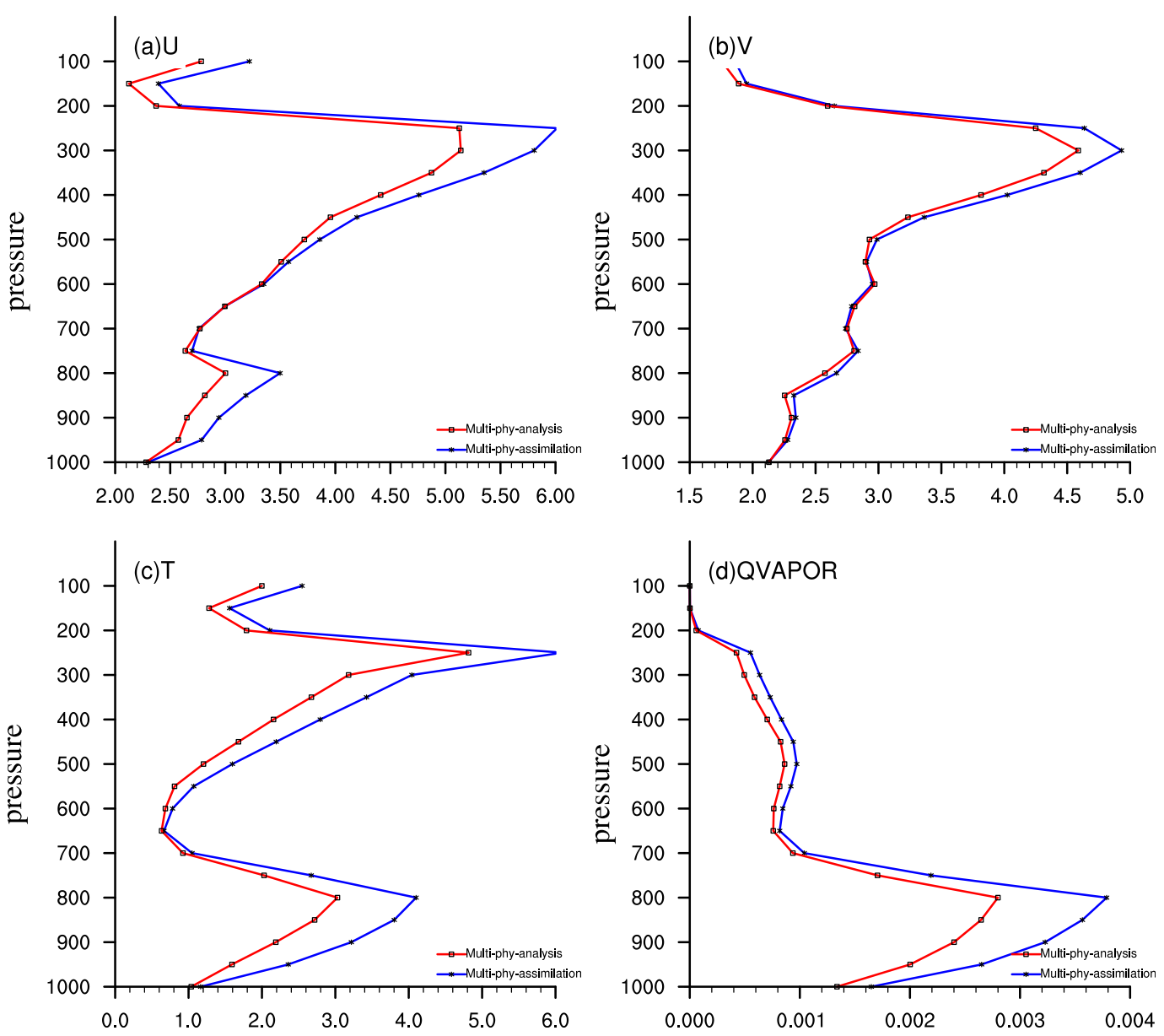

FIG. 13. Domain-averaged vertical profiles of the RMSEs in (a) zonal wind $\left(\mathrm{m} \mathrm{s}^{-1}\right)$, (b) meridional wind $\left(\mathrm{m} \mathrm{s}^{-1}\right)$, (c) temperature (K), and (d) water vapor mixing ratio $\left(\mathrm{kg} \mathrm{kg}^{-1}\right)$ at 1900 UTC 8 Dec 2015 from the Multiphyassimilation (blue) and the Multiphy-analysis (red) experiments.

the ensemble four-dimensional variational data assimilation by the radar data observation operator and ultimately lead to significant variations in the assimilation and prediction results.

4) To obtain the optimal results for the assimilation and prediction, three methods based on the physical ensemble technique, which can weaken the influence of the parameterization schemes, are designed. It is found that precipitation forecasts of the assimilation experiments that use the physical ensemble method are noticeably improved compared with those of the CNTL experiment, but different physical ensemble methods lead to different precipitation forecasts. Among the three assimilation experiments, Multiphy-forecast resulted in the most accurate simulated precipitation, followed by Multiphy-analysis, and finally Multiphy-assimilation.

5) Differences in simulated precipitation between Multiphy-assimilation and Multiphy-analysis are primarily attributed to differences in the initial fields caused by the use of different physical ensemble methods. The analysis field of the Multiphyanalysis experiment is much closer to the simulated "true" atmosphere than that of Multiphy-assimilation, resulting in more accurate simulated precipitation. Unlike Multiphy-analysis, which averages the initial fields before the numerical forecast, Multiphy-forecast averages the results after the numerical forecasts, thus taking into account the uncertainty of the initial field. This effectively filters out variations that occur during the numerical forecast, leading Multiphy-forecast to achieve the highest accuracy. This also indicates that even if an advanced assimilation method improves the accuracy of the initial fields, uncertainty still exists, and that an effective method of reducing this uncertainty is the ensemble forecast. 
Acknowledgments. This research is supported by the BJG Open Research Fund for NJCAR (NJCAR2018ZD03) and the National Nature Science Foundation of China under Grant 41375063.

\section{REFERENCES}

Buehner, M., 2005: Ensemble-derived stationary and flowdependent background-error covariances: Evaluation in a quasi-operational NWP setting. Quart. J. Roy. Meteor. Soc., 131, 1013-1043, https://doi.org/10.1256/qj.04.15.

— , P. L. Houtekamer, C. Charette, H. Mitchell, and B. He, 2010a: Intercomparison of variational data assimilation and the ensemble Kalman filter for global deterministic NWP. Part I: Description and single-observation experiments. Mon. Wea. Rev., 138, 1550-1566, https://doi.org/10.1175/ 2009MWR3157.1.

,,,,---- and,$- 2010 \mathrm{~b}$ : Intercomparison of variational data assimilation and the ensemble Kalman filter for global deterministic NWP. Part II: One-month experiments with real observations. Mon. Wea. Rev., 138, 1567-1586, https:// doi.org/10.1175/2009MWR3158.1.

Cossu, F., and K. Hocke, 2014: Influence of microphysical schemes on atmospheric water in the Weather Research and Forecasting Model. Geosci. Model Dev., 7, 147-160, https://doi.org/ 10.5194/gmd-7-147-2014.

Courtier, P., J.-N. Thépaut, and A. Hollingsworth, 1994: A strategy for operational implementation of 4D-Var, using an incremental approach. Quart. J. Roy. Meteor. Soc., 120, 13671387, https://doi.org/10.1002/qj.49712051912.

Dudhia, J., 1989: Numerical study of convection observed during the Winter Monsoon Experiment using a mesoscale twodimensional model. J. Atmos. Sci., 46, 3077-3107, https:// doi.org/10.1175/1520-0469(1989)046<3077:NSOCOD >2.0.CO;2.

Ek, M. B., K. E. Mitchell, Y. Lin, E. Rogers, P. Grunmann, V. Koren, G. Gayno, and J. D. Tarpley, 2003: Implementation of Noah land surface model advances in the National Centers for Environmental Prediction operational mesoscale Eta Model. J. Geophys. Res., 108, 8851, https://doi.org/10.1029/ 2002JD003296.

Gao, J., M. Xue, A. Shapiro, and K. K. Droegemeier, 1999: A variational method for the analysis of three-dimensional wind fields from two Doppler radars. Mon. Wea. Rev., 127, 2128-2142, https://doi.org/10.1175/1520-0493(1999)127<2128: AVMFTA $>2.0 . \mathrm{CO} ; 2$.

Grell, G. A., and S. R. Freitas, 2014: A scale and aerosol aware stochastic convective parameterization for weather and air quality modeling. Atmos. Chem. Phys., 14, 5233-5250, https:// doi.org/10.5194/acp-14-5233-2014.

Hamill, T. M., and C. Snyder, 2000: A hybrid ensemble Kalman filter-3D variational analysis scheme. Mon. Wea. Rev., 128, 2905-2919, https://doi.org/10.1175/1520-0493(2000)128<2905: $\mathrm{AHEKFV}>2.0 . \mathrm{CO} ; 2$.

Hong, S.-Y., and J.-O. J. Lim, 2006: The WRF single-moment 6-class microphysics scheme (WSM6). J. Korean Meteor. Soc., 42, 129-151.

—_, Y. Noh, and J. Dudhia, 2006: A new vertical diffusion package with an explicit treatment of entrainment processes. Mon. Wea. Rev., 134, 2318-2341, https://doi.org/10.1175/MWR3199.1.

Houtekamer, P. L., and H. L. Mitchell, 2005: Ensemble Kalman filtering. Quart. J. Roy. Meteor. Soc., 131, 3269-3289, https:// doi.org/10.1256/qj.05.135.
Jankov, I., W. A. Gallus Jr., M. Segal, B. Shaw, and S. E. Koch, 2005: The impact of different WRF Model physical parameterizations and their interactions on warm season MCS rainfall. Wea. Forecasting, 20, 1048-1060, https://doi.org/10.1175/ WAF888.1.

Lan, W., J. Zhu, M. Xue, T. Lei, and J. Gao, 2010: Storm-scale ensemble Kalman filter data assimilation experiments using simulated Doppler radar data. Part II: Imperfect model tests (in Chinese). J. Atmos. Sci., 34, 737-753.

Liu, C., Q. Xiao, and B. Wang, 2008: An ensemble-based fourdimensional variational data assimilation scheme. Part I: Technical formulation and preliminary test. Mon. Wea. Rev., 136, 3363-3373, https://doi.org/10.1175/2008MWR2312.1.

Lorenz, E. N., 1963: Deterministic nonperiodic flow. J. Atmos. Sci., 20, 130-141, https://doi.org/10.1175/1520-0469(1963) $020<0130: \mathrm{DNF}>2.0 . \mathrm{CO} ; 2$.

Ma, X., X. Lu, Y. Yu, J. Zhu, and J. Chen, 2014: Progress on hybrid ensemble-variational data assimilation in numerical weather prediction (in Chinese). J. Trop. Meteor., 30, 1188-1195.

Meng, Z., and F. Zhang, 2007: Tests of an ensemble Kalman filter for mesoscale and regional-scale data assimilation. Part II Imperfect model experiments. Mon. Wea. Rev., 135, 14031423, https://doi.org/10.1175/MWR3352.1.

Mlawer, E. J., S. J. Taubman, P. D. Brown, M. J. Iacono, and S. A. Clough, 1997: Radiative transfer for inhomogeneous atmospheres: RRTM, a validated k-correlated model for the longwave. J. Geophys. Res., 102, 16 663-16 682, https://doi.org/ 10.1029/97JD00237.

Peffers, L., 2011: Hybrid variational ensemble data assimilation with initial condition and model physics uncertainty. M.S. thesis, Dept. of Earth, Ocean and Atmospheric Sciences, Florida State University, 111 pp., http://diginole.lib.fsu.edu/ islandora/object/fsu\%3A180297.

Qiu, C., and J. Chou, 2006: Four-dimensional data assimilation method based on SVD: Theoretical aspect. Theor. Appl. Climatol., 83, 51-57, https://doi.org/10.1007/s00704-005-0162-z.

Skamarock, W. C., and Coauthors, 2008: A description of the Advanced Research WRF version 3. NCAR Tech. Note NCAR/TN-475+STR, 113 pp., http://dx.doi.org/10.5065/ D68S4MVH.

Snyder, C., and F. Zhang, 2003: Assimilation of simulated Doppler radar observations with an ensemble Kalman filter. Mon. Wea. Rev., 131, 1663-1677, https://doi.org/10.1175//2555.1.

Sun, J., and N. A. Crook, 1997: Dynamical and microphysical retrieval from Doppler radar observations using a cloud model and its adjoint. Part I: Model development and simulated data experiments. J. Atmos. Sci., 54, 1642-1661, https://doi.org/10.1175/1520-0469(1997)054<1642:DAMRFD> 2.0.CO;2.

— , and - 1998: Dynamical and microphysical retrieval from Doppler radar observations using a cloud model and its adjoint. Part II: Retrieval experiments of an observed Florida convective storm. J. Atmos. Sci., 55, 835-852, https://doi.org/ 10.1175/1520-0469(1998)055<0835:DAMRFD > 2.0.CO;2.

Tian, X., and X. Feng, 2015: A non-linear least squares enhanced POD-4DVar algorithm for data assimilation. Tellus, 67A, 25340, https://doi.org/10.3402/tellusa.v67.25340.

_ Z. Z. Xie, and A. Dai, 2008: An ensemble-based explicit fourdimensional variational assimilation method. J. Geophys Res., 113, D21124, https://doi.org/10.1029/2008JD010358.

,,--- C. Shi, B. Jia, F. Chen, and K. Yang, 2009: A dualpass variational data assimilation framework for estimating soil moisture profiles from AMSR-E microwave brightness 
temperature. J. Geophys. Res., 114, D16102, https://doi.org/ 10.1029/2008JD011600.

,,--- B. Jia, and C. Shi, 2010: A microwave land data assimilation system: Scheme and preliminary evaluation over China. J. Geophys. Res., 115, D21113, https://doi.org/10.1029/ 2010JD014370.

-,- , and Q. Sun, 2011: A POD-based ensemble fourdimensional variational assimilation. Tellus, 63A , 805-816, https://doi.org/10.1111/j.1600-0870.2011.00529.x.

_ _ - Y. Liu, Z. Cai, Y. Fu, H. Zhang, and L. Feng, 2014 A joint data assimilation system (Tan-Tracker) to simultaneously estimate surface $\mathrm{CO}_{2}$ fluxes and 3-D atmospheric concentrations from observations. Atmos. Chem. Phys., 14, 13 281-13 293, https://doi.org/10.5194/acp-14-13281-2014.

Wang, B., J. Liu, S. Wang, W. Cheng, L. Juan, C. Liu, Q. Xiao, and Y.-H. Kuo, 2010: An economical approach to four-dimensional variational data assimilation. Adv. Atmos. Sci., 27, 715-727, https://doi.org/10.1007/s00376-009-9122-3.

Wang, H., T. Auligné, and H. Morrison, 2012: Impact of microphysics scheme complexity on the propagation of initial perturbations. Mon. Wea. Rev., 140, 2287-2296, https://doi.org/ 10.1175/MWR-D-12-00005.1.
Wernli, H., M. Paulat, M. Hagen, and C. Frei, 2008: SAL-A novel quality measure for the verification of quantitative precipitation. Mon. Wea. Rev., 136, 4470-4487, https://doi.org/10.1175/ 2008MWR2415.1.

Xiao, Q., Y. Kuo, J. Sun, W. Lee, D. Barker, and E. Lim, 2007: An approach of radar reflectivity data assimilation and its assessment with the inland QPF of Typhoon Rusa (2002) at landfall. J. Appl. Meteor. Climatol., 46, 14-22, https://doi.org/10.1175/ JAM2439.1.

Yang, Y., L. Zhang, and S. Xie, 2016: Casual analysis of an unusual torrential rain in Guangdong in December 2015 (in Chinese). Torrential Rain Disaster, 35, 326-333.

Zhang, B., X. Tian, J. Sun, C. Feng, Y. Zhang, L. Zhang, and S. Fu, 2015: PODEn4DVar-based radar data assimilation scheme: Formulation and preliminary results from real-data experiments with Advanced Research WRF (ARW). Tellus, 67A, 26045, https://doi.org/10.3402/tellusa.v67.26045.

Zhang, F., Y. Weng, J. A. Sippel, Z. Meng, and C. H. Bishop, 2009: Cloud-resolving hurricane initialization and prediction through assimilation of Doppler radar observations with an ensemble Kalman filter. Mon. Wea. Rev., 137, 2105-2125, https://doi.org/ 10.1175/2009MWR2645.1. 